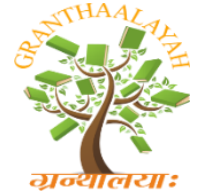

INTERNATIONAL JOURNAL OF RESEARCH GRANTHAALAYAH A knowledge Repository

RACSIT - 17

\title{
SURVEY ON POWER AWARE LOAD BALANCING IN CLOUD COMPUTING
}

\author{
Manjula *1, Madhu B R ${ }^{2}$ \\ ${ }^{* 1} 4^{\text {th }}$ Semester CSE, M.Tech. Student, SET, Jain University, Bengaluru, India \\ ${ }^{2}$ Associate Professor, SET, Jain University, Bengaluru, India
}

DOI: https://doi.org/10.29121/granthaalayah.v5.i4RACSIT.2017.3358

\begin{abstract}
Cloud computing in the current years has been taking its development from the logical to the non logical and business applications. Control utilization and Load adjusting are essential and complex issue in computational Cloud. Load Balancing is an imperative segment in the item benefits based cloud computing. There is a need to create calculations that can catch this multifaceted nature yet can be effortlessly actualized and used to fathom an extensive variety of load adjusting situations in a Data and Computing escalated applications. Because of increment in the quantity of server farms, which relate to electrical vitality cost, crest control scattering, cooling and carbon emanation. These days, control utilization is one of the significant issues for the operation and upkeep of server farms. Vitality costs for server farms are multiplying like clockwork and have effectively crossed $\$ 19$ billion. Be that as it may, a lot of this power is squandered as servers are for the most part sit. Sit without moving servers can likewise expend as much as $60 \%$ of pinnacle power utilization. To measure the energy consumed by processor, and we call these are a load balancing techniques, we introduced many algorithm like Power Aware Load balancing (PALB), Double Threshold- Power Aware Load balancing (DT-PALB), Dynamic Round-Robin (DRR), Equally Spread Active Execution( ESCE ), Minimum Cost Maximum flow (MCMF). In this paper we compare algorithm and we will conclude which algorithm is best for power aware load balancing in cloud.
\end{abstract}

Keywords: Load Balancing; PALB; DPALB; Cloud Computing; Power Aware; Data Center.

Cite This Article: Manjula, and Madhu B R. (2017). "SURVEY ON POWER AWARE LOAD BALANCING IN CLOUD COMPUTING." International Journal of Research - Granthaalayah, 5(4) RACSIT, 81-85. https://doi.org/10.29121/granthaalayah.v5.i4RACSIT.2017.3358.

\section{Introduction}

Distributed computing gets its name as a relationship for the Internet. Generally, the Internet is symbolized in framework diagrams as a cloud. The expression "cloud" systematized from the universe of communicate correspondences when supplier began using virtual private framework 
(VPN) organizations for data exchanges. distributed computing basically infers Internet Computing, broadly the Internet is seen as a conglomeration of fogs. Thusly, distributed computing is laid out as using the web to give development engaged organizations to the overall public and affiliations. As indicated by NIST(National Institute of Standards and Technology), cloud computing is a model for empowering advantageous, on request organize door to a common gathering of configurable registering assets.

Stack adjusting is one of the focal issues in distributed computing. It is a section that spreads the dynamic neighborhood workload comparatively over every one of the middle focuses in the entire cloud to maintain a strategic distance from circumstance where two or three focus focuses are over whelmingly stacked while others are sit still or doing little work. It completes a high consumer loyalty and asset use degree, along these lines enhancing the general execution and asset utility of the structure. Cutting down the centrality utilization of server farms is an attempting and complex issue since figuring applications and information are developing so rapidly that immovably more noteworthy servers and plates are depended upon to process them enough quick inside the required time. This is principal for guaranteeing that the future progression of Cloud figuring is conceivable. Conveyed registering with consistently unavoidable frontend customer gadgets cooperating with back-end server farms will understand a colossal accelerating of the hugeness use. To address this issue and drive Green Cloud figuring, server develop assets should be regulated in a centrality practical way. Specifically, Cloud assets should be assigned not exclusively to fulfill Quality of Service (QoS) necessities directed by clients by techniques for Benefit Level Agreements (SLAs), besides to decreasing vitality utilize.

We propose an observational examination of load changing structures like PALB estimation, the power mind full stack balancer, PALB, keeps up the power utilization of all open figure focus focuses besides, courses virtual machines in a way that is power convincing. The objective of PALB is to keep up high openness of enroll focus assets while decreasing the Total power ate up by the cloud. Also, another calculation DT-PALB (Double Edge Energy Aware Load Balancing) number keeps up the condition of all figure focus focuses, likewise, in light of use rates, picks the measure of process focuses that ought to work. the DRR is, the basic lead Avoid adding additional virtual machines to a leaving physical machine. The second control stimulates the mix procedure and draws in Dynamic Round-Robin to shutdown physical machines. Furthermore, ESCE is, The sporadic affirmation based scattered issue round robin. Choice rely on upon minimum load. MCMF is, It depends on upon a made diagram delineation out of the dynamic asset movement issue.

This paper is further divided into following sections. Section II discusses related work. In section III Comparative Analysis Section, IV contains Conclusion, and Section V References.

\section{Related Work}

Investigate has been performed by on load altering of virtual machines. There has additionally been much research in towards various styles of load modifying, for example, the three-level approach. Another approach endorsed instead of limiting force, there ought to be choices to pick specific power or execution choices per work. Different techniques for part association of oblige and essentialness have been proposed and considered. The CloudSim gadget is utilized for 
driving tests on the power watchful datacenter. Importance association in Data Centers is a champion among the most troublesome issues confronted by the foundation suppliers. Because of the well-ordered increase in the data and dealing with, it is unrealistic to keep control over the power usage in the course required as the execution will basically be impacted. Dynamic RoundRobin[1] strategy is a development to the Round-Robin technique. it utilizes two picks that assistance to mix virtual machines. The crucial pick says that if a virtual machine has completed and still other virtual machines that are supported on the same physical machine, then this physical machine can't perceive any new virtual machine. Such physical machines are called "leaving" state physical machines, that recommends when the distinctive virtual machines complete their execution, by then we can shut down this physical machine. The second pick says that if there is a "leaving" state physical machine that is utilized for drawn out stretch out of time, then as confined to sitting tight for that virtual machines to complete, the physical machine is compelled to move the diverse virtual machines to other physical machines, and from that point shutdown the physical machine after the improvement wraps up. The edge holding up time is tended to by the "retirement edge". A physical machine will be compelled to move to all the virtual machines and a brief timeframe later close it down as it is in the leaving state however after beyond what many would consider possible, it couldn't complete all other virtual machines These two gages are utilized by the Dynamic Round-Robin framework with a particular ultimate objective to solidify virtual machines executed by the Round-Robin strategy. As appeared by the fundamental direct, adding additional virtual machines to a leaving physical machine is maintained a strategic distance from. As indicated by the second control, the blend methodology swing out to be fast and it empowers Dynamic Round-Robin to shutdown physical machines, with the end target that the measure of physical machine used to run each virtual machine is lessened, in this manner the power can be spared. The PALB tally [2] has three straightforward areas. The changing section is totally in charge of the assertion of the virtual machines that will be displayed. It at first accumulates the rate of usage of every dynamic PC focus. In the event that if, all method focus focuses are more than $75 \%$ utilize, then PALB starts another virtual machine as the PC focus having the most irrelevant utilize. At the point exactly when all the PC base focuses are on $75 \%$ use, then all the open PC focus focuses are working. Something else, the booting of the new virtual machine (VM) on the PC focus having the most lifted use is required (essentially on the off chance that it can oblige the measure of the VM). Right when $25 \%$ of the advantages are accessible, then as of late the edge estimation of $75 \%$ of utilization was picked, no shy of what one virtual machine could be obliged by the utilization of 12 out of 20 open blueprints. The going with upscale area of this check is utilized for power on extra PC focus focuses (if there are different accessible PC focus focuses). This is done if all at show dynamic PC focus focuses have use more than $75 \%$. The downscale part is in charge of shutting down sit out of contraption enroll focus focuses. In the event that the PC focus focuses are having under $25 \%$ utilization of its favorable circumstances, PALB gives a shutdown summon to that specific focus point. ESCE algorithm[3], The estimation of the business measure by the cloud boss and after that checking for the for the accessibility of the virtual machine and in addition the limit of the virtual machine. Once the open asset (virtual machine) measure and the level of the business matches, then immediately the occupation scheduler scatters saw virtual machine or asset for the occupation in a line. The effect of the ESCE algorithm[3] is that a change is found in the reaction time and the arranging time. The proportionate scattering of employments is done, $\mathrm{n} \mathrm{o} \mathrm{w}$ the entire enrolling structure is stack adjusted and there is no such virtual machines that are underutilized. Thusly realness, there is a diminishment in the cost of 

virtual machine and besides the cost of information exchange. This depends on upon the adjusted Bin-Packing model[4] that experiences adaptability issues with different cases in like manner, increment in the measure of PMs and furthermore the deals. This has induced for finding a substitute differentiating alternative to the dynamic asset situation issue and along these lines prompts to the Minimum Cost Maximum Flow (MCMF) figuring. The DT-PALB count [5] has three essential bits. The altering section is responsible for understanding where virtual machines will be instantiated. It does this by at first gathering the utilization rate of every dynamic figure focus. For the situation that all figure focus focuses $\mathrm{n}$ are over $75 \%$ use, PALB instantiates another virtual machine on the technique focus with the most diminished use number. It legitimizes deciding for the condition where all figure focus focuses are over $75 \%$ use, a large portion of the accessible enroll focus focuses are in operation. Something else, the new virtual machine (VM) is booted on the enroll focus with the most essential use (on the off chance that it can oblige the measure of the VM). On the off chance that any middle having usage in the midst of $75 \%$ and $25 \%$ then find most underutilized focus call attention to dole out vm to that inside. In the event that any middle point having under $25 \%$ use then move vm to other focus point. The purpose of imprisonment of $75 \%$ use was picked since when $25 \%$ of the preferences are open, no shy of what one more virtual machine can be suited utilizing three out of five open arrangements. The upscale segment of the calculation is utilized to control on extra figure focus focuses (the length of there are more open enroll focus focuses). It does this if affirm directly capable figure focus focuses have use more than $75 \%$. The downscale bit is responsible for shutting down sit out of contraption process focuses. In case the strategy focus point is utilizing under $25 \%$ of its advantages, PALB sends a shutdown demand to that inside.

\section{Comparative Analysis}

\begin{tabular}{|c|c|c|}
\hline Algorithm & Description & Energy Saved \\
\hline $\begin{array}{l}\text { Power Aware Load } \\
\text { balancing } \\
\text { (PALB) }\end{array}$ & $\begin{array}{l}\text { Keeps up the condition of all } \\
\text { process } \\
\text { hubs, and in light of use rate, } \\
\text { chooses the number of register }\end{array}$ & $75 \%$ power utilized \\
\hline $\begin{array}{l}\text { Double Threshold- Power } \\
\text { Aware Load balancing } \\
\text { (DT-PALB) }\end{array}$ & $\begin{array}{l}\text { one of the fundamental } \\
\text { contributions of } \\
\text { the bunch controller is load } \\
\text { adjusting } \\
\text { figure hubs. }\end{array}$ & $\begin{array}{l}\text { all computenodes } n \\
\text { are } \\
\text { above } 90 \% \text { utilization }\end{array}$ \\
\hline $\begin{array}{l}\text { Dynamic Round- Robin } \\
\text { (DRR) }\end{array}$ & $\begin{array}{l}\text { The first run the show Maintain a } \\
\text { strategic distance fromincluding } \\
\text { a dditional virtual machines to a } \\
\text { resigning physical ma chine. } \\
\text { The second run the show speeds } \\
\text { up the consolidationprepare } \\
\text { furthermore, empowers Dynamic } \\
\text { Round- Robin to shutdown } \\
\text { physicalmachine. }\end{array}$ & $80 \%$ power is utilized \\
\hline $\begin{array}{l}\text { Equally Spread Active } \\
\text { Execution(ESCE) }\end{array}$ & $\begin{array}{l}\text { The arbitrary choice based } \\
\text { appropriated issue round robin. } \\
\text { Choice depend on slightest } \\
\text { stack. }\end{array}$ & $25 \%$ power utilized \\
\hline $\begin{array}{l}\text { Munmum cost Maxmin } \\
\text { Flow(MCMF) }\end{array}$ & $\begin{array}{l}\text { It is in view of A directed diagram } \\
\text { speak to representation of the } \\
\text { Dynamic Asset distribution Issue. }\end{array}$ & $\begin{array}{l}55 \% \text { poweris } \\
\text { utilized }\end{array}$ \\
\hline
\end{tabular}




\section{Conclusion}

Distributed computing has for the most part been gotten by the business or affiliation however there are many existing issues like Load Balancing, Virtual Machine Consolidation, Energy Management, et cetera. We propose DT-PALB strategy to pass on the virtual machines for power saving reason. DTPALB is an increase from one of a kind PALB. The DT-PALB we proposed can reduce the amount of drive on physical machine and typical power usage appear differently in relation to other send computations with power saving. Furthermore, the connection between our figuring's and the Round Robin booking computations in other cloud demonstrates basic change in saving power. Our Power Aware Load Balancing count, PALB, keeps up the state of all procedure center points, and in perspective of use rates, picks the amount of enlist center points that should work. Existing Load Balancing strategies that have been thought about, generally focus on diminishing overhead, advantage response time and upgrading execution et cetera., and a bit of the techniques have considered the essentialness use factors. In this manner, there is a need to develop an Energy-careful load modifying system that can upgrade the execution of distributed computing nearby most extraordinary resource utilize, hence lessening imperativeness usage.

\section{References}

[1] Jeffrey M. Galloway, Karl L. Smith, Susan S. Vrbsky, Power Aware Load Balancing for Cloud Computing, Vol I WCECS 2011, October 19-21, 2011.

[2] Jeffrey Galloway, Karl Smith, Jeffrey Carver. An Empirical Study of Power Aware Load Balancing in Local Cloud Architectures. 978-0-7695-4654-4/12 \$26.00 2012 IEEE

[3] Mr. Jayant Adhikari, Prof. Sulabha Patil, Tulsiramji Gaikwad-Patil, Double Threshold Energy Aware Load Balancing In Cloud Computing, IEEE-2013.

[4] Sankari Subbiah1, Varalakshmi Perumal, Power Aware Resource Optimization in Cloud, 2013 IEEE.

[5] Karanpreet Kaur, Ashima Narang, Kuldeep Kaur, Load Balancing Techniques of Cloud Computing, IJMCR volume-3 2013.

[6] Garima Joshi, S. K. Verma. A Review on Load Balancing Approach in Cloud Computing. International Journal of Computer Applications (0975 - 8887) Volume 119 - No.20, June 2015

*Corresponding author.

E-mail address: manjulagungwar@gmail.com 